\title{
Formación Docente desde un Enfoque Inclusivo. A 25 Años de la Declaración de Salamanca, Nuevos y Viejos Desafíos
}

\section{Teacher Education from an Inclusive Approach. 25 Years after the Salamanca Statement, New and Old Challenges}

\author{
Cynthia Duk ${ }^{1 *}$ \\ Tatiana Cisternas ${ }^{2}$ \\ Liliana Ramos ${ }^{3}$ \\ ${ }^{1}$ Universidad Central de Chile, Chile \\ ${ }^{2}$ Universidad Alberto Hurtado, Chile \\ ${ }^{3}$ Universidad Diego Portales, Chile
}

\begin{abstract}
Este artículo desarrolla un análisis sobre la formación docente desde un enfoque inclusivo y su relación con la perspectiva y aportes de la Declaración de Salamanca. En una primera parte se analiza la influencia y vigencia de los cambios que propone la Declaración en torno a las políticas, las escuelas, los docentes y su formación. En este marco, se examinan las recientes políticas educativas desde el punto de vista de las demandas y desafíos para la formación del profesorado de todos los niveles educativos y disciplinas. Se constata la importancia de desarrollar en los docentes competencias para el abordaje de la diversidad en el aula y se revisan, desde la literatura internacional, tres propuestas para responder a la pregunta sobre cuáles son las capacidades que debe promover la formación inicial docente para encarnar los dilemas y principios de la inclusión educativa. Finalmente, a partir de todo el análisis realizado, las autoras proponen cuatro competencias fundamentales para la formación de todos los profesores y ofrecen recomendaciones para las políticas de formación inicial docente.
\end{abstract}

Descriptores: Formación docente; Educación inclusiva; Competencias del docente; Valores; Actitudes.

\begin{abstract}
This article develops an analysis of teacher education from an inclusive approach and its relationship with the perspective and contributions of the Salamanca Declaration. The first part describes the changes required by the Declaration for the policies, the school organization, its teachers and the authority of its vision. With this framework, we analyze how recent educational policies involve new challenges for the teacher education and teachers of all educational levels and disciplines. These demands show that teachers need to develop competencies oriented to address diversity in the classroom. Consequently, three proposals are reviewed from international literature, to answer the question about what are the capacities that should be promoted by initial teacher education to embody the dilemmas and principles of inclusive education. Finally, from all the analysis carried out, the authors propose four minimum competencies for the training of all teachers and offer recommendations for educational policies.
\end{abstract}

Keywords: Teacher education; Inclusive education; Teachers skills; Values; Attitudes. 


\title{
1. La declaración de Salamanca y su influencia sobre las políticas educativas en Chile
}

A 25 años de la Declaración Salamanca (DdS) sobre Necesidades Educativas Especiales, NEE (UNESCO, 1994), es posible afirmar que su mayor mérito radica en que consiguió posicionar a nivel internacional el enfoque de educación inclusiva e impulsó el desarrollo de cambios en los sistemas educativos y las políticas, manteniendo la vigencia de sus planteamientos a lo largo de estos años.

Su principal legado es que reforzó el objetivo de Educación para Todos de Jontiem (1990), con un foco particular en los grupos más vulnerables a la exclusión, la marginación y discriminación, poniendo en el horizonte por primera vez la aspiración a una escuela inclusiva, al señalar que:

\begin{abstract}
El principio rector de este Marco de Acción es que las escuelas deben acoger a todos los niños, independientemente de sus condiciones fisicas, intelectuales, sociales, emocionales, lingüísticas u otras". $\Upsilon$ añade que, "el mérito de las escuelas "integradoras", no es sólo que sean capaces de dar una educación de calidad a todos los niños; con su creación se da un paso muy importante para intentar cambiar las actitudes de discriminación, crear comunidades que acojan a todos y sociedades integradoras ${ }^{l}$.
\end{abstract}

Sin lugar a dudas, el mensaje de Salamanca ha tenido gran influencia siendo recogido y reforzado con distintos matices en importantes foros, convenciones e instrumentos de derechos humanos que han marcado la agenda de los países e impactado las legislaciones y políticas nacionales (Duk y Murillo, 2018). A tal punto, que el compromiso asumido por los Estados en el marco de la Agenda 2030 de Desarrollo Sostenible, es "Garantizar una educación inclusiva, equitativa y de calidad, y promover oportunidades de aprendizaje durante toda la vida para todos" (ODS4; ONU, 2015).

A modo de contextualización, mientras se celebraba la Conferencia de Salamanca, Chile se encontraba en pleno proceso de transición a la democracia tras 17 años de dictadura militar. La década del 90 en el país se caracterizó por grandes expectativas y prometedoras reformas educativas que buscaban recuperar el sentido democrático de la educación, generar condiciones que permitieran ampliar la cobertura hasta lograr acceso universal y mejorar la calidad de los procesos y resultados educativos que se encontraban muy descendidos, especialmente en los sectores más desfavorecidos.

En el ámbito de la Educación Especial, un hito digno de mencionar es la promulgación del Decreto 490/1990 de Integración Escolar, el cual abre el camino para que estudiantes con discapacidad pudiesen escolarizarse en la escuela regular con apoyos especializados, a través de distintas opciones de integración que combinaban espacios y tiempos de enseñanza entre el aula común y el aula especial o sala de recursos, según el tipo y grado de déficit de los estudiantes y su capacidad de adaptación social. Este modelo predominó durante toda la década y se expandió a nivel nacional a través de los llamados "Proyectos de Integración”, impulsados y financiados con recursos desde la Educación Especial.

En este contexto histórico, los compromisos y recomendaciones de Jontiem (1990) y Salamanca (1994) supusieron un importante impulso para la agenda transformadora de la

${ }^{1}$ Cabe señalar que el término de "escuelas integradoras" equivale a escuelas inclusivas, en la versión en inglés de la Declaración se utiliza "inclusive school". Se trata entonces de una diferencia en la traducción. 
época en Chile. En 1996 el Ministerio de Educación en colaboración con OREALC/UNESCO organizan la primera reunión regional de seguimiento de su Marco de Acción para los países de América Latina y el Caribe. En la ocasión, se puso de manifiesto la necesidad imperiosa de sensibilizar y capacitar a los docentes con prioridad en los de educación básica, por considerarse que no estaban preparados para integrar a estudiantes con discapacidad.

En el caso de Chile, se promovieron acciones y programas puntuales de capacitación docente en distintas temáticas relacionadas con la atención a las NEE en contextos de integrados, una demanda que se acrecentaba en la medida que se ampliaba la cobertura de integración en el sistema educativo regular, alcanzando en la actualidad a cerca del $60 \%$ de las escuelas y liceos del país.

Con el paso del tiempo, se produce un cambio de visión desde la integración a la educación inclusiva que implica una perspectiva más amplia, que abarca a todos los/as estudiantes bajo el reconocimiento del derecho que todos tienen de recibir una educación de calidad bajo condiciones de equidad. Desde esta visión la inclusión tiende a ser asumida como un asunto de la educación general que trasciende y no se limita a la educación especial. Será en la primera década del milenio cuando comienza a asentarse esta idea y la inclusión es concebida como un principio que debe guiar las políticas y las prácticas educativas. No obstante, estos avances a nivel de las definiciones de política, cohabitan hasta hoy la lógica de la integración junto con el nuevo paradigma de la inclusión, con las consecuentes repercusiones en las actuaciones educativas.

Producto de esta evolución, la preocupación deja de estar centrada en la inclusión de determinadas poblaciones y se traslada al contexto, enfocándose en cómo crear entornos educativos más inclusivos, donde todos los/as estudiantes se sientan acogidos y valorados. Este cambio de perspectiva interpela a los docentes a desarrollar capacidades que les permitan ofrecer respuestas educativas efectivas y pertinentes a la diversidad de estudiantes, que aseguren la participación y aprendizaje de todos.

\section{La formación docente como eslabón para avanzar hacia escuelas inclusivas. Un desafío complejo e insoslayable}

Según lo anteriormente señalado, es posible deducir que una escuela inclusiva como la que se propone, y de la cual Salamanca fue precursora, requiere de cambios sustantivos en la formación inicial y continua de los docentes, siendo un factor clave para avanzar en la dirección de una educación de mayor calidad y equitativa para todos. Al respecto, resulta interesante observar cómo el año 1994 Salamanca se anticipa apelando a los gobiernos a reorientar la formación docente, de manera que todo profesor tenga algún nivel de preparación para dar respuesta a las NEE en las escuelas comunes: "garantizar que, en un contex to de cambio sistemático, los programas de formación del profesorado, tanto inicial como continua, estén orientados a atender las NEE en las escuelas integradoras" (p. 9).

Más específicamente, en las recomendaciones del Marco de Acción la DdS plantea que "Los programas de formación inicial deberán inculcar en todos los profesores tanto de primaria como de secundaria, una orientación positiva hacia la discapacidad (...). Los 
conocimientos y las aptitudes requeridos son básicamente los de una buena pedagogía..." (p. 31).

En este sentido, junto con reconocer que son los docentes y los directores escolares una pieza clave para la inclusión, aunque no la única, resalta el papel fundamental que tiene la formación docente inicial y continua para el éxito de las transformaciones que se requieren y, por tanto, la responsabilidad que le cabe a las instituciones de educación superior y a los Ministerios de Educación para asegurar que dichos procesos se materialicen: "A las universidades corresponde un importante papel consultivo (...), en particular en relación con la investigación, la evaluación, la preparación de formadores de profesores y la elaboración de programas y materiales pedagógicos" (p. 33).

Más allá de Salamanca, la preocupación por la formación de docentes de todos los niveles y modalidades, como condición indispensable para el desarrollo de un sistema educativo inclusivo, se instaló en el país en buena parte a consecuencia de la presión de las escuelas y sus docentes frente a la creciente presencia de estudiantes con NEE en los distintos niveles de la trayectoria educativa. Así también, debido a un mayor reconocimiento de los pueblos originarios, el aumento de estudiantes de otras nacionalidades y culturas a propósito del fenómeno de la migración, y la aparición de otros temas relevantes antes invisibilizados, por ejemplo, la inclusión de la comunidad LGTBI, que desde luego en su conjunto representan importantes retos para la formación docente inicial y continua.

Al respecto, las preguntas que siguen rondando en torno a la formación docente, dicen relación con: (i) el tipo de perfil docente que se requiere formar para la inclusión, (ii) las competencias profesionales que se necesitan desarrollar, (iii) las capacidades que habría que instalar en el sistema para abordarlas, (iv) el modelo curricular y los contenidos que serían más pertinentes para alcanzar el perfil docente deseado.

A la luz de lo expuesto, el presente trabajo busca contribuir a la reflexión en torno a la preparación de todos los profesores de cara a la inclusión, recogiendo los sentidos de la DdS en esta materia, y en articulación con las demandas que emergen de las políticas educativas, los cambios logrados y los desafíos que siguen pendientes para impulsar y sostener el proceso de renovación de la formación inicial docente.

\section{Políticas educativas y desafíos para la formación inicial docente en inclusión educativa}

En el marco de los aportes y la vigencia de la Declaración de Salamanca, a continuación se revisan diversos instrumentos y normativas promulgadas entre 2009 y 2018, que promueven la inclusión, identificando los desafíos específicos que se desprenden de ellos para la formación docente.

\subsection{Ley General de Educación: Hacia un sistema educativo inclusivo}

La Ley General de Educación (LGE) (20.370/ 2009), marca un hito relevante en la orientación inclusiva que ha ido incorporando el sistema educativo chileno en la última década. Este instrumento reconoce que el sistema educativo se fundamenta en los derechos garantizados en la Constitución, así como en tratados internacionales ratificados por Chile que se encuentren vigentes entre los cuales se encuentra la Convención sobre Derechos de Personas con Discapacidad (2006). 
Varios principios que inspiran la LGE se orientan a la construcción de un sistema educativo inclusivo, entre ellos: (i) Calidad de la educación, que implica asegurar que todas/os los estudiantes logren los objetivos generales y estándares de aprendizaje, independientemente de sus condiciones y circunstancias; (ii) Equidad del sistema educativo, referido al aseguramiento de la igualdad de oportunidades de todas/os en cuanto a recibir una educación de calidad, con especial atención en quienes requieren apoyo especial; (v) Integración, referido a la incorporación de estudiantes de diversas condiciones sociales, religiosas, económicas y culturales; (vi) e Interculturalidad, que insta a reconocer y valorar a los/as estudiantes, considerando su especificidad cultural y de origen, lengua, cosmovisión e historia (Art. 3).

Respecto de las NEE, la ley establece que "se entenderá que un alumno presenta necesidades educativas especiales cuando precisa ayudas y recursos adicionales, ya sean humanos, materiales o pedagógicos, para conducir su proceso de desarrollo y aprendizaje, y contribuir al logro de los fines de la educación" (Art. 23). Así, este instrumento privilegia un enfoque educativo y de apoyo para comprender las dificultades de aprendizaje por sobre el modelo centrado en el déficit (Ministerio de Educación, 2009a).

En este marco, la LGE plantea desafíos significativos para la formación inicial de profesoras y profesores:

- Formar a los/as futuros docentes en los tratados internacionales ratificados por Chile que promueven la inclusión educativa, comprendiendo el enfoque de derechos humanos en que se sustentan.

- Abordar el análisis de los principios de la ley, promoviendo la comprensión de su orientación inclusiva como marco de acción para los/as docentes.

- Favorecer la comprensión del concepto de NEE desde un enfoque educativo y no centrado en déficits.

\subsection{Decreto 170: Los programas de integración escolar como medida de respuesta a las NEE}

En Chile la principal estrategia promovida por el Ministerio de Educación, desde inicios de los 90, para la educación de los estudiantes con NEE en las escuelas regulares y la provisión de recursos de apoyo, son los Programas de Integración Escolar (PIE). En este contex to, el Decreto 170/2009 establece normas y mecanismos que permiten la asignación de la subvención de educación especial a los estudiantes identificados con NEE (permanentes o transitorias). Con este propósito, regula requisitos, procedimientos y el perfil de los/as profesionales idóneos que intervienen en los procesos evaluativos para determinar los/as estudiantes con NEE y en consecuencia los apoyos que requieren. Define las funciones de los profesionales apoyo y promueve el trabajo colaborativo entre docentes de educación regular y especial, de preferencia dentro del aula común, estableciendo asignación de horas semanales para el trabajo conjunto de planificación, evaluación y seguimiento del aprendizaje, involucrando la participación de la familia (Ministerio de Educación, 2009b).

Desde una perspectiva inclusiva, esta normativa avanza por dos razones principales: (i) establece que la evaluación diagnóstica ha de ser integral, interdisciplinaria y contextualizada y considerar información proveniente de los docentes, familia y del propio estudiante según corresponda; (ii) promueve el trabajo colaborativo entre profesionales y la participación en instancias de perfeccionamiento con el fin de fortalecer la respuesta 
educativa a la diversidad en el aula (Art. 86). No obstante, estos avances, a su vez esta política lleva a clasificar a los estudiantes en función de ciertas categorías diagnósticas más propias del ámbito de la salud, coexistiendo en la misma normativa dos enfoques contrapuestos, el enfoque médico y el enfoque pedagógico para dar respuesta a las NEE. Esto obedece a la necesidad de identificar a los estudiantes destinatarios de recursos adicionales, como consecuencia del modelo de subvención a la demanda que existe en el país, sistema que ha potenciado el "etiquetaje", llegando incluso al sobrediagnóstico, con los consabidos efectos no deseados para los estudiantes, sus familias y el sistema.

Algunos desafíos para la formación inicial docente que se desprenden de esta política son:

- Comprender el fenómeno de la discapacidad desde una perspectiva multidimensional basada en el enfoque biopsicosocial y desarrollar competencias para la identificación y evaluación oportuna de las NEE, desde un enfoque educativo y curricular.

- Desarrollar la capacidad para reflexionar críticamente en torno a dilemas asociados a la inclusión y a las tensiones derivadas de las contradicciones que persisten a nivel de enfoques, políticas y prácticas.

- Promover el desarrollo de competencias comunicativas, cognitivas y socioemocionales requeridas para trabajar en contextos de colaboración, que favorezcan el aprendizaje y la participación de todas/os los estudiantes.

- Favorecer el uso diversas medidas pedagógicas, estrategias de aprendizaje y de apoyo que permitan tomar decisiones que favorezcan el aprendizaje de todas/os, considerado sus diferencias.

\subsection{Ley de inclusión escolar: Elimina mecanismos del sistema que generan desigualdad y exclusión en la educación.}

La ley 20.845/2015, más conocida como Ley de Inclusión Escolar, (Ministerio de Educación, 2015a) introduce regulaciones que intentan contrarrestar el modelo de educación de mercado imperante en el país, reducir la segregación escolar y asegurar mayor igualdad de oportunidades para todos. Con este propósito, instaura transformaciones en relación a tres aspectos críticos que generan barreras a la inclusión en escuelas subvencionadas con fondos públicos: pone fin a la selección de estudiantes al ingreso, prohíbe el lucro en la educación y elimina el copago proveniente de las familias. Uno de sus principios es el de Integración e Inclusión, que hace referencia a la eliminación de toda forma de discriminación arbitraria que afecte el aprendizaje y participación de los estudiantes, definiendo la escuela como "un lugar de encuentro entre los/as estudiantes de distintas condiciones socioeconómicas, culturales, étnicas, de género, de nacionalidad o religión” (Art. 3).

Respecto de los/as estudiantes que presentan NEE, esta ley plantea que el Estado tiene el deber de asegurar una educación inclusiva de calidad a todas las personas, y de generar condiciones para el acceso y permanencia de los/as estudiantes con NEE en establecimientos de educación regular o especial, según sea el interés superior del niño o niña (Art. 4).

Para la formación inicial docente refuerza el desafío instalado por la LGE relativo a cultivar los principios que están a la base de un sistema educativo inclusivo. De igual forma, al tratarse de un instrumento que regula la educación escolar general, se avanza en la 
comprensión de la inclusión como un asunto que no compete exclusivamente a la educación especial. En este sentido, no solo considera las NEE como las variables que determinan contextos de diversidad, sino que amplía la compresión a otras muchas diferencias que determinan la construcción de procesos de identidad y alteridad en las y los estudiantes.

\subsection{Decreto 83: Diversificación de la enseñanza y adecuaciones curriculares}

El Decreto 83/2015 establece criterios y orientaciones de adecuación curricular específicamente para los niveles de educación parvularia (inicial) y básica. Las medidas de flexibilización curricular que propone, para hacer más pertinente y accesible el currículo, son aplicables a las escuelas educación regular, incluyendo la modalidad de adultos, y a las escuelas especiales y hospitalarias.

Desde la perspectiva inclusiva, esta normativa implica un avance principalmente por dos razones: (i) promueve la diversificación de la enseñanza y los principios de diseño universal para el aprendizaje como estrategia para dar respuesta a la diversidad de todas/os los estudiantes; (ii) establece como referente para la educación de los estudiantes con discapacidad, las bases curriculares nacionales con adecuaciones en la medida que se requiera, derogando el uso de programas de estudio diferenciados por déficits (Ministerio de Educación, 2015b).

Algunos desafíos para la formación docente que se pueden visualizar a partir de este decreto son:

- Brindar una formación pedagógica, con énfasis en el desarrollo curricular y la didáctica, que considere las necesidades educativas individuales, diversos ritmos $\mathrm{y}$ formas de aprender de los/as estudiantes.

- Manejar el principio de accesibilidad y diseño universal, y sus diversas formas de aplicación en el ámbito educativo.

- Desarrollar la capacidad para tomar decisiones de adecuación curricular de forma colaborativa, considerando la situación de los/as estudiantes y sus contextos.

\subsection{Decreto 67: Más allá de la calificación, evaluación para el aprendizaje}

El decreto 67/2018 de evaluación, que entrará en vigencia el 2020, establece normas mínimas sobre evaluación, calificación y promoción en los niveles de educación básica y media. Este decreto asume la evaluación como parte intrínseca del proceso de enseñanza aprendizaje, valorando tanto su uso formativo como sumativo para la toma de decisiones (Ministerio de Educación, 2018). Este instrumento insta a las escuelas a implementar medidas de diversificación en las actividades de aprendizaje y de evaluación, así como adecuaciones curriculares, en sintonía con los decretos 170 y 83.

Como desafíos para la formación inicial docente, podemos enunciar los siguientes:

- Fortalecer en la formación los enfoques de evaluación formativa y autentica ofreciendo un repertorio amplio de procedimientos e instrumentos aplicables en la práctica.

- Desarrollar la capacidad para planificar e implementar procesos evaluativos diversificados, que tengan en cuenta los diferentes niveles de comprensión y formas expresión de los estudiantes. 
Finalmente, señalar que los desafíos para la formación docente asociados a las políticas que orientan la educación inclusiva en Chile, se vinculan con las propuestas del Marco de Acción de Salamanca, en particular con aquellas que señalan: (i) inculcar una orientación positiva hacia la discapacidad, (ii) desarrollar la capacidad para evaluar necesidades especiales y adaptar el currículum, (iii) individualizar procedimientos pedagógicos para responder a un mayor número de aptitudes, (iv) trabajar colaborativamente con otros profesionales y con las familias.

\section{Desarrollo de capacidades en la formación inicial de profesores para abordar los desafíos de una educación inclusiva}

Uno de los principales desafíos que enfrentan los sistemas educativos en la actualidad es lograr la igualdad entre los seres humanos respetando al mismo tiempo la diversidad. Tal como ha sido descrito hasta ahora, a propósito de la DdS, la educación inclusiva se ha configurado como una respuesta para avanzar hacia el reconocimiento y respeto de las diferencias de origen socioeconómico, culturales, étnicas, de orientación sexual e identidad de género, discapacidad, y los diferentes talentos con que las y los estudiantes enfrentan su proceso formativo, proporcionando una respuesta educativa de calidad y pertinente a sus singularidades.

Desde la mirada de distintos actores, experiencias, investigaciones y organismos internacionales, existe consenso respecto de la necesidad de contar con profesores mejor preparados para responder a la diversidad y promover la participación y aprendizaje de todos los/as estudiantes. Ello supone preparar a los futuros docentes transitando desde una mirada compensatoria hacia una aproximación que traslada el desafío a la institución escolar y sus barreras para el aprendizaje y la participación.

Informes recientes que recogen la experiencia internacional en torno a la formación inicial docente en este ámbito, advierten la falta de estudios empíricos sobre las prácticas efectivas de formación inicial que apunten al desarrollo de profesionales orientados hacia la inclusión. Asimismo, este diagnóstico señala que tenemos poca información acerca de cómo los futuros docentes cambian sus perspectivas para volverse "maestros orientados a la inclusión”. Es escasa la evidencia empírica sobre qué iniciativas son efectivas para preparar a los formadores de docentes para la diversidad (European Commission, 2017).

La DdS remarca la necesidad de una formación para que "todos" los y las docentes desarrollen una "buena pedagogía" acorde a las necesidades de todos sus estudiantes, en particular aquellos que enfrentan mayores barreras o se encuentran en riesgo de exclusión. Ello supone que "todo profesor, al margen del nivel educativo en el que se desempeñe, de su especialidad o de la asignatura que imparta debe estar formado para trabajar con diversidad de estudiantes y en contextos diversos" (Duk, 2014, p. 63).

Ahora bien, ¿qué competencias necesita desarrollar la formación inicial de todos los docentes para abordar estos desafíos? Resulta pertinente responder a esta pregunta primero a partir de la propuesta vigente en Chile para la formación inicial en este ámbito. En nuestro país, contamos con un marco orientador para la formación inicial de todos los 
profesores de enseñanza básica y media en torno al trabajo con la diversidad ${ }^{2}$. Los estándares pedagógicos para la formación en educación básica y media reconocen la necesidad de preparar a los futuros docentes para responder a la diversidad en el aula señalando que: El futuro profesor o profesora comprende que la educación es un derecho de todos los estudiantes y que la diversidad es una fuente de riqueza para el desarrollo y aprendizaje de las comunidades educativas. Por ello, está preparado para diseñar, implementar y evaluar estrategias pedagógicas que contribuyan a hacer efectiva la igualdad de oportunidades y evitar la discriminación. Lo que se manifiesta cuando:

- Respeta a cada uno de los estudiantes, sus familias y sus comunidades, y actúa previniendo el efecto discriminatorio que pueden tener sus propias acciones, decisiones y juicios respecto a las características personales derivadas de variables sociales, sexuales, étnicas, de apariencia física o variables de aprendizaje en el desarrollo académico, afectivo y social de los jóvenes y adolescentes que estarán a su cargo.

- Conoce los requerimientos pedagógicos de los estudiantes con necesidades educativas especiales, incluidos los estudiantes con talentos especiales, y evalúa con otros profesores cuándo es necesario adaptar su enseñanza a estos requerimientos, realizar un trabajo coordinado con especialistas o derivar a otras instancias.

- Conoce la normativa relativa a integración de la discapacidad en el sistema educativo.

- Conoce estrategias para favorecer la inclusión e integración de los estudiantes, seleccionando recursos pedagógicos apropiados para estimular el desarrollo de sus fortalezas y respectivas autonomías (MINEDUC, 2014, p. 74).

En esta misma línea, algunos estudios concluyen que una barrera importante para la inclusión es la percepción de los docentes que no se sienten preparados ni con suficientes recursos para trabajar con todos los/as estudiantes en contextos de diversidad y atender las necesidades educativas especiales (González-Gil et al, 2016; Tenorio, 2011 en Castillo Armijo y Miranda, 2018; Cisternas y Lobos 2019). Considerando lo reciente de los cambios de paradigma y el giro desde un enfoque segregador o integrador hacia una perspectiva inclusiva a continuación, describimos tres propuestas de competencias docentes que tienen el valor de sostenerse en evidencia disponible, ser pertinentes a los actuales desafíos y contar con una sólida base conceptual en materia de educación inclusiva.

\subsection{Propuesta de un Perfil del docente para la educación inclusiva}

El Proyecto de Formación del profesorado para la educación inclusiva liderado en 2012 por la Agencia Europea para las Necesidades Especiales y la Educación Inclusiva (en adelante Agencia), sostiene que la preparación de todos los docentes en esta línea contribuiría a enfrentar diversos retos sociales y políticos: enfrentar las desventajas educativas producto de las inequidades sociales, abordar problemas derivados de la pobreza y marginalización, hacer frente al abandono escolar, mejorar los logros educativos y las competencias necesarias para la participación en la sociedad y romper las barreras

${ }^{2}$ Cabe señalar que en el marco de la Ley 20.903 promulgada el año 2016 se ha dispuesto renovar los estándares pedagógicos y disciplinares para todas las carreras de pedagogía del país, los cuales entrarían en vigencia prontamente. 
que experimentan los estudiantes con discapacidad. Luego de un trabajo realizado a lo largo de tres años ${ }^{3}$, se concluye que formar para estos desafíos supone articular el desarrollo, no sólo de conocimientos y capacidades docentes, sino también la promoción de valores y actitudes profesionales.

De este modo, la Agencia estructura un perfil profesional basado en cuatro valores fundamentales a partir de los cuales se articulan actitudes, creencias, conocimientos y habilidades: a) respetar la diversidad de todos los estudiantes, en tanto ésta es un recurso y un valor educativo; b) favorecer a todos los estudiantes, con altas expectativas para todos ellos; c) Trabajar colaborativamente; d) Desarrollo y aprendizaje permanente del docente para abordar los desafíos de la enseñanza.

Para cada una de estas cuatro dimensiones hemos seleccionado actitudes, conocimientos y habilidades que nos parecen clave y orientadoras para su abordaje en los programas de formación inicial de profesores en nuestro país a la luz de los desafíos expuestos en apartados anteriores (Agencia Europea para el Desarrollo de la Educación del Alumnado con Necesidades Educativas Especiales, 2012):

\subsubsection{Capacidad para valorar en positivo la diversidad de los estudiantes}

Concepciones de la educación inclusiva

- Los/as futuros docentes analizan y construyen actitudes y creencias sobre la educación inclusiva: se fundamenta en la igualdad, en los derechos humanos y en la democracia; creen en la participación de todos los estudiantes implicados en actividades de aprendizaje significativas.

- Conocen los principios teóricos y prácticos que estructuran la educación inclusiva; que ésta se dirige a todos los estudiantes y no sólo para quienes presentan necesidades de apoyo o están en riesgo de exclusión; conciben la educación inclusiva como la presencia, participación y aprendizaje de todos los estudiantes.

- Desarrollan la capacidad de examinar de manera crítica sus creencias y el impacto en la práctica.

El punto de vista del docente ante las diferencias entre estudiantes

- Los futuros docentes desarrollan comportamientos y creencias sobre lo "normal" y lo "diferente"; asumen que la diversidad de los estudiantes es un recurso que amplía oportunidades para aprender.

- Conocen las características propias de la diversidad de los estudiantes; comprenden que se aprende de formas diferentes y esto se utiliza como un apoyo; saben que la escuela en tanto comunidad influye en la autoestima y el aprendizaje de los estudiantes.

- Desarrollan la habilidad de aprender de las diferencias de sus estudiantes y de visibilizar la diversidad cuando aborda el currículo.

Cabe señalar que las orientaciones formuladas por Durán y Giné (2011) coinciden ampliamente con estas capacidades. Relevan la importancia de promover capacidades para

${ }^{3}$ Proyecto de la Agencia en el que participaron más de cincuenta expertos de veinticinco países vinculados a al diseño de políticas y a la formación de profesores tanto de educación especial como general. 
la aceptación de todos los/as estudiantes como propios; un sólido conocimiento sobre las diferencias de los alumnos; aprender a colaborar con otros docentes y profesionales; manejar variedad de estrategias para la inclusión que les ayuden a tomar decisiones curriculares y metodológicas que faciliten el mayor grado de participación y aprendizaje de todos; y contar con herramientas de investigación-acción para reflexionar y trasformar los entornos y la propia práctica (pág.159-160).

\subsubsection{Capacidad para apoyar a todos los estudiantes}

Promover el aprendizaje académico, práctico, social y emocional de todos los estudiantes

- Los futuros docentes desarrollan la convicción de que el aprendizaje es principalmente una actividad social; creen que las expectativas de los docentes son determinantes en el aprendizaje del estudiante.

- Aprenden sobre las formas de desarrollo típicos y atípicos de los estudiantes y comprenden y comparan diferentes modelos de aprendizaje.

- Tienen habilidad para desarrollar capacidades comunicativas en sus estudiantes, saben formar estudiantes autónomos; implementar enfoques de trabajo cooperativo entre pares y usar estrategias de evaluación que atienda a los aprendizajes conceptuales, sociales y emocionales.

Enfoques pedagógicos eficientes en aulas heterogéneas

- Los/as futuros docentes creen que es su responsabilidad facilitar el aprendizaje de todos los estudiantes; creen en el potencial de estos para desarrollarse y aprender;

- Desarrollan conocimientos sobre los procesos de aprendizaje y los modelos de enseñanza que los promueven; conocen formas de organizar el aula para generar ambientes positivos; conocen enfoques de enseñanza individualizada; manejan estrategias para dar respuesta a las barreras para el aprendizaje; comprenden la evaluación formativa y los modos de diferenciar los contenidos curriculares, los procesos de aprendizaje y los recursos de enseñanza.

- Desarrollan habilidad para utilizar los contenidos curriculares como instrumento inclusivo; aprenden a diferenciar estrategias de enseñanza, contenidos y resultados de aprendizaje; saben cómo facilitar el aprendizaje cooperativo entre pares; utilizar un repertorio diverso de estrategias de enseñanza, e implementar enfoques de evaluación y retroalimentación formativa evitando catalogar a los estudiantes.

\subsubsection{Capacidad para trabajar en equipo}

Trabajar con las familias

- Los futuros docentes muestran respeto por las raíces culturales, sociales y las opiniones de las familias; también desarrollan una actitud de comunicación y colaboración eficaz con las familias como parte de su responsabilidad docente.

- Comprenden que la enseñanza se basa en el trabajo en equipo y desarrollan la habilidad de comprometer a las familias en el aprendizaje de sus hijos, y comunicarse adecuadamente con todas ellas más allá de sus diferencias culturales, étnicas, lingüísticas o sociales. 
Trabajar con diversos profesionales de la educación

- Los futuros docentes desarrollan actitudes que valoran la colaboración, el compañerismo y el trabajo en equipo y creen que esta forma de trabajo contribuye al desarrollo profesional.

- Conocen los beneficios del trabajo en equipo y diversos modelos de colaboración. Desarrollan un lenguaje y perspectivas básicas sobre el trabajo en equipo.

- Demuestran habilidades de liderazgo y de resolución de problemas en colaboración con otros.

Como se puede constatar, es interesante en esta propuesta cómo cada dimensión se compone de un conjunto de actitudes, conocimientos y habilidades. La premisa es que las actitudes necesitan un nivel de conocimiento o comprensión y éstos también se articulan con las habilidades para enfrentar una situación práctica.

\subsubsection{Desarrollo profesional y personal}

Docentes son profesionales que deben reflexionar

- Los futuros docentes creen que la enseñanza requiere planificación sistemática, evaluación, reflexión y cambio permanente; asumen una práctica reflexiva para su trabajo con otros y para revisar sus propias decisiones.

- Reconocen las destrezas para aprender a aprender y para poner en acción la reflexión; conocen estrategias para la evaluación de su trabajo y el de otros; saben sobre métodos de investigación y cómo utilizarlos en el trabajo docente.

- Desarrollan la habilidad para evaluar sistemáticamente el trabajo realizado e involucrar a otros profesionales en la reflexión sobre la docencia y el aprendizaje.

Iniciar con la FID un aprendizaje y desarrollo profesional que es continuo

- Los futuros docentes creen en la importancia y necesidad de un desarrollo profesional constante; muestran actitud de apertura para aprender y transformar su práctica porque asumen que la docencia es una actividad de aprendizaje continuo.

- Conocen el marco normativo y las responsabilidades de la profesión que éste involucra

- Desarrollan habilidades como la flexibilidad, apertura y proactividad para innovar y desarrollarse profesionalmente, además aprenden a contribuir en el desarrollo de la comunidad educativa.

Desde su creación, este marco ha tenido fuerte influencia en buena parte de los países que participaron en su construcción, así como en algunos países latinoamericanos (Echeita, 2012). A la luz de los desafíos de nuestro país, es una propuesta que puede orientar los currículos de formación y perfiles de egreso.

\subsection{Competencias docentes para la enseñanza en contextos de diversidad sociocultural}

Este marco de competencias busca ofrecer posibilidades para mejorar la formación de profesores respecto al manejo de la diversidad en las salas de clases. Es producto del trabajo colectivo de diversos países pertenecientes al Council of Europe project on Policies 
and Practices for Teaching Sociocultural Diversity (2009). Participaron especialistas de ocho países ${ }^{4}$, quienes durante tres años trabajaron en el desarrollo un marco que define 18 competencias básicas para trabajar con la diversidad en el aula. Para sus autores, la competencia docente para la enseñanza de la diversidad sociocultural se refiere al "qué", "cómo" y "por qué" y se organiza en tres dimensiones: a) conocimiento y comprensión; b) comunicación y relación con los otros y c) gestión del aula y la enseñanza. Cabe señalar que se asume la enseñanza como una práctica social que exige más que simples estrategias, técnicas o conocimiento disciplinar. Enseñar requiere resolver situaciones complejas en entornos diversos (Arnesen, Allen y Simonsen, 2009).

\subsubsection{Dimension: Conocimiento y Compresión}

El conocimiento y la comprensión se consideran requisitos que permiten a los docentes ser sensibles y responder eficazmente a la diversidad. Se basan en una visión del conocimiento como proceso reflexivo, crítico y en desarrollo.

- Conocimiento y comprensión de las políticas y normativas relacionadas con la diversidad

- Conocimiento acerca de los marcos conceptuales vigentes y comprensión de principios clave respecto de la diversidad en educación

- Conocimiento sobre las diferentes dimensiones de la diversidad: cultural, género, necesidades educativas especiales y comprender sus implicancias en los entornos escolares.

- Conocimiento de un rango (repertorio) de aproximaciones de la enseñanza, estrategias y materiales para responder a la diversidad.

- Habilidad para indagar en torno a temáticas/asuntos referidos a las diferencias socioculturales

- Reflexión sobre su propia identidad y compromiso con la diversidad

4.2.2. Dimension: Comunicación y relación con los otros

Estas competencias están en el corazón del compromiso y respuesta de los docentes a la diversidad. Es el espacio en que se crean condiciones para que el aula y la escuela sean inclusivas y donde construyen relaciones basadas en la confianza y el respeto mutuo.

- Comunicación positiva y significativa con estudiantes, padres, docentes y otros profesionales asumiendo la existencia de diferencias en las formas de aproximarse, con distintas culturas y experiencias

- Reconocimiento y respuesta a dimensiones culturales y comunicativas de el o los lenguajes utilizados en la escuela

- Propiciar apertura y respeto en la comunidad escolar

- Incentivar a los estudiantes para comprometerse en el aprendizaje individualmente y en cooperación con otros

- Involucrar a todos los padres y apoderados en actividades escolares y en la toma de decisiones colectiva

${ }^{4}$ Participan Austria, Bulgaria, Chipre, Estonia, Francia, Grecia, Noruega e Inglaterra. 
- Mediar los conflictos y la violencia para prevenir exclusión y fracaso escolar 4.2.3. Dimension: Gestión del aula y enseñanza

Esta área de competencia involucra acciones para crear un ambiente de aprendizaje solidario y seguro, con interacción social positiva y participación activa en el aprendizaje. Se busca construir una cultura organizacional cooperativa y no discriminatoria que materialice la idea de convivir y aprender juntos.

- Abordar la diversidad en el currículo y en el desarrollo institucional (de la comunidad escolar)

- Establecer un entorno de aprendizaje participativo, inclusivo y seguro

- Seleccionar y modificar las estrategias de enseñanza de acuerdo a las necesidades de aprendizaje de los estudiantes

- Analizar críticamente el papel de la diversidad en materiales de apoyo a la enseñanza

- Utilizar una variedad de enfoques para desarrollar prácticas de enseñanza y evaluación que sean culturalmente sensibles a la diversidad.

- Reflexión y evaluación sistemática de la propia práctica y el impacto de ésta en sus estudiantes

Este marco se construye con especial interés en la formación inicial de profesores para responder a la diversidad sociocultural. Se reconoce que la forma en que las y los docentes estén preparados para comprometerse con la diversidad en su sentido más amplio es de crucial importancia. De allí que resulte altamente interesante para los contextos escolares actuales de migración e interculturalidad, que caracterizan a nuestro sistema educativo.

\subsection{Capacidades para el desarrollo de una pedagogía inclusiva}

El Proyecto para las Prácticas Inclusivas, liderado por Lani Florian y desarrollado en Escocia en la Universidad de Aberdeen entre los años 2006 y 2010, aporta y complementa las propuestas anteriores y tiene el valor de configurarse como una experiencia que ha sido implementada y estudiada en su impacto. Se proponen tres principios para estructurar los contenidos y experiencias de formación inicial docente que se describen en el siguiente cuadro: (Florian y Black-Hawkins, 2011; Florian y Spratt, 2013).

\subsubsection{Comprender el aprendizaje y la diferencia}

Los docentes deben comprender la diferencia como un aspecto esencial al desarrollo humano. Supone reemplazar visiones deterministas del aprendizaje y de la capacidad de cada estudiante basadas en una concepción tradicional de inteligencia hacia una perspectiva que cree en la modificabilidad y el progreso de todos los niños, niñas, adolescentes y jóvenes.

\subsubsection{Asumir la inclusión como como un asunto de justicia social}

Los docentes deben comprometerse con el aprendizaje de todos sus estudiantes, asumiendo que los problemas para aprender son desafíos de la enseñanza y no dificultades o carencias de los alumnos. De este modo, la formación de los docentes se debe orientar hacia prácticas donde se amplían los recursos para que todos participen y aprendan, rechazando pedagogías que mantienen una lógica de enseñanza para la mayoría combinada con enseñanza focalizada en un grupo específico. 


\subsubsection{Convertirse en un profesional activo}

Se espera que los docentes comprendan la profesión como parte de un desarrollo continuo que se construye a través del trabajo con otros. Un profesional activo, es decir, capacitado para transformar e innovar sus prácticas para apoyar el aprendizaje de todos sus estudiantes. Para ello, el futuro/a docente está preparado para trabajar colaborativamente en la búsqueda de impulsar la participación y asegurar el aprendizaje.

Diversos autores coinciden en que es fundamental desde el punto de vista valórico, que la formación inicial docente fortalezca los principios de justicia social imbricados en el concepto de inclusión (Cisternas y Lobos, 2019; Durán y Giné, 2011, Hirmas y Ramos 2013; Simón y Echeita, 2013). Asimismo, el perfil que describe esta propuesta se articula con lo que plantea Rosa Blanco (2005) respecto de las capacidades que requiere un docente y con ello la formación inicial que reciba:

Se requiere ante todo un docente que se atreva a asumir riesgos y pruebe nuevas formas
de enseñanza, que reflexione sobre su práctica para transformarla, que valore las
diferencias como elemento de enriquecimiento profesional y que sea capaz de trabajar
en colaboración con otros docentes, profesionales y familias. Un docente que
personalice las experiencias comunes de aprendizaje, es decir: que conozca bien a todos
sus alumnos y sea capaz de diversificar y adaptar el currículo; que plantee diferentes
situaciones y actividades de aprendizaje; que ofrezca múltiples oportunidades; que
tenga altas expectativas respecto al aprendizaje de todos sus alumnos y les brinde el
apoyo que precisan... (Blanco, 2005, p. 176)

\section{Conclusiones: Recomendaciones para la formación docente en inclusión}

Las conclusiones de este trabajo articulan y recogen los aportes de estas iniciativas, reportes y experiencias internacionales.

\section{Cuatro competencias para la formación inicial de todos los docentes}

Tomado en cuenta los planteamientos de la DdS, los desafíos para la formación docente detectados a la luz de las políticas vigentes, las propuestas internacionales revisadas respecto de las competencias que debe demostrar un docente con una orientación inclusiva, y las características del contexto educativo chileno, consideramos fundamental que la formación inicial de todo profesor/a ofrezca oportunidades para:

Valorar positivamente la diversidad como oportunidad y recurso para el aprendizaje de todos/as desde el enfoque de derechos

Esto significa comprender el aporte que cada miembro de la comunidad educativa realiza, desde su propia identidad y diferencia, en beneficio del aprendizaje, participación y progreso en la trayectoria educativa de todas/os los estudiantes. A su vez, supone reconocer y visibilizar la diversidad cultural, lingüística, religiosa, de género y formas de aprendizaje, entre otras, para que cada estudiante se sienta valorado por ser quien es, promoviendo su sentimiento de pertenencia y fortaleciendo la construcción de su identidad personal en una comunidad segura, acogedora y libre de discriminación. Esta competencia requiere formar la capacidad reflexiva y de análisis crítico del futuro/a docente, para proponer e implementar experiencias que permitan aprovechar la diversidad en beneficio del aprendizaje de todos/as, evaluando los resultados y ajustando su actuación en una perspectiva de mejora constante de la propia práctica. 
Demostrar capacidad y disposición para trabajar colaborativamente con distintos actores para el desarrollo de una comunidad educativa inclusiva

Los/as futuros docentes deben aprender a trabajar en colaboración con otros, entre los que se incluyen otros docentes, profesionales de apoyo, familias, directivos; y establecer redes con la comunidad. La capacidad y disposición para trabajar colaborativamente resulta esencial para enfrentar contextos complejos y diversos, que no admiten respuestas únicas y uniformes; en este sentido, desde la colaboración se podrán movilizar, gestionar e implementar los apoyos que algunas/os estudiantes requieren para avanzar en su trayectoria educativa. Esta competencia debe considerar como marco de acción los valores inclusivos, que constituyen el fundamento ético para que las y los docentes puedan desempeñarse, tanto en comunidades educativas que avanzan hacia la inclusión como en otras que aún mantienen prácticas de exclusión.

Tomar decisiones de flexibilización y diversificación curricular ampliando las oportunidades de aprendizaje de todas y todos los estudiantes

Flexibilizar o diversificar la enseñanza es una competencia fundamental que debe poseer un docente orientado hacia la inclusión. A partir de una concepción de aprendizaje y desarrollo no determinista, se espera que los/as futuros docentes sean capaces de conocer, utilizando diversos procedimientos, los intereses, formas de aprender y necesidades educativas de sus estudiantes, para brindarles distintas oportunidades de aprendizaje que favorezcan su autonomía y capacidad de elección, fortaleciendo la participación y el compromiso con el aprendizaje individual y colectivo. En este proceso de toma de decisiones, es relevante monitorear los aprendizajes y evaluar la necesidad de modificar aspectos de la propuesta pedagógica. Para esto, se requiere el manejo de un amplio repertorio de recursos y estrategias didácticas que abran oportunidades para que los/as estudiantes pongan en juego sus hipótesis y construyan progresivamente nuevos aprendizajes en espacios de colaboración con sus pares.

Generar entornos de aprendizaje inclusivos que promuevan una convivencia respetuosa de la diversidad

Es fundamental que los/as futuros docentes desarrollen capacidades para responder a las necesidades socioemocionales de sus estudiantes, propiciando ambientes de aula que permitan la participación y el aprendizaje de todas/os. Lo anterior supone el manejo de herramientas para promover una convivencia en que se respeten las diferencias, se aborden pacíficamente las situaciones de conflicto, se tomen medidas preventivas para reducir situaciones de violencia y discriminación y se utilice el currículo como una oportunidad para educar en formas de relación cotidianas que apunten al logro de una cultura escolar y convivencia fundada en valores democráticos e inclusivos, al servicio del bienestar de todos los/as estudiantes.

\section{Recomendaciones para las políticas de formación docente}

Sin duda, las instituciones formadoras de profesores y sus académicos tienen un papel decisivo en el desarrollo de las competencias requeridas para la formación desde el enfoque inclusivo, la renovación de los modelos formativos y de los planes de estudio de las diferentes carreras educación frente a las nuevas exigencias del sistema educativo. Sin desmerecer los esfuerzos y avances logrados a nivel de las IES, las evidencias muestran que los cambios en la formación de los docentes han sido muy discretos de cara a las 
transformaciones que exige el enfoque inclusivo a nivel de las concepciones, actitudes y prácticas pedagógicas.

A modo de cierre se exponen a continuación algunas de las recomendaciones relativas a la formación docente, que se desprenden de las Propuestas para Avanzar Hacia un Sistema Educativo Inclusivo en Chile, emanadas de la Mesa Técnica de Educación Especial convocada por el Ministerio de Educación en el marco de la Reforma Educacional (2014):

- La inclusión y la respuesta a la diversidad deben constituirse en un eje transversal que permee la formación pedagógica de todos los docentes, sea cual sea el nivel educativo en el que se van a desempeñar o disciplina que impartan.

- En tanto función de todos los docentes, el tema de la inclusión y la diversidad en sus distintas expresiones debe incorporarse como contenido en los planes de estudio y en las mallas curriculares de todas las carreras de educación. Para ello se propone una estructura curricular que contemple un tronco común de formación pedagógica, que promueva el desarrollo de competencias para la inclusión y la atención a la diversidad, y que esta formación se imparta de preferencia en cursos combinados que incluyan estudiantes de las distintas carreras de educación, favoreciendo el trabajo interdisciplinario y colaborativo entre los futuros docentes.

- La formación inicial debe estar alineada con las políticas educativas, el currículum nacional y estar estrechamente vinculada a la práctica. Es necesario que los futuros docentes a lo largo de su trayectoria formativa tengan acceso a prácticas profesionales guiadas en distintos tipos de instituciones y contextos educativos.

- Los estándares establecidos para la formación docente inicial de los distintos niveles educativos y disciplinas deben estar alineados con los principios de inclusión y promover el desarrollo de perfiles de egreso coherentes con este enfoque.

- Consecuente con los principios de inclusión, las IES deben contar con políticas y medidas de acción afirmativa que faciliten el acceso, permanencia y egreso de estudiantes con discapacidad, estableciendo sistemas de apoyo y acompañamiento a los estudiantes y docentes. Asimismo, dentro de las medidas de acción afirmativa las instituciones de educación superior debieran incorporar al cuerpo académico formadores representativos de la diversidad.

Finalmente, tal como fue reconocido en la DdS, el profesorado es y seguirá siendo una figura clave para la transformación de la cultura y las prácticas educativas en una perspectiva inclusiva. Asumir la diversidad en sus distintas expresiones como una oportunidad formativa y diseñar experiencias de aprendizaje que beneficien a todos los estudiantes, promoviendo su participación y sentimiento de pertenencia a la comunidad escolar, en un clima de convivencia respetuoso de las diferencias y con sentido de justicia social, es una tarea de gran envergadura y complejidad, que requiere de nuevas competencias docentes y, en consecuencia, formadores capacitados para promoverlas.

\section{Referencias}

Arnesen, A., Allen, J. y Simonsen, E. (2009). Policies and practices for teaching socio-cultural diversity. Concepts, principles and challenges in teacher education. Estrasburgo: Council of Europe. 
Blanco, R. (2005). Los docentes y el desarrollo de escuelas inclusivas, Revista PRELAC, 1, 174-177.

Cisternas, T. y Lobos, A. (2019). Profesores nóveles de enseñanza básica: Dilemas, estrategias y obstáculos para abordar los desafíos de una educación inclusiva. Revista Latinoamericana de Educación Inclusiva, 13(1), 37-53. https://doi.org/10.4067/S0718-73782019000100037

Duk, C. (2014). La formación y el desarrollo profesional de los docentes para una educación inclusiva. En A. Marchesi, R. Blanco y L. Hernández (Coords.), Avances y desafíos de la educación inclusiva en Iberoamérica (pp. 61-70). Madrid: OEI.

Duk, C. y Murillo, F. J. (2018). El mensaje de la educación inclusiva es simple, pero su puesta en práctica es compleja. Revista Latinoamericana de Educación Inclusiva, 12(1), 11-13. https://doi.org/10.4067/So718-73782018000100011

Durán, D. y Giné, C. (2011). La formación del profesorado para la educación inclusiva: Un proceso de desarrollo profesional y de mejora de los centros para atender la diversidad. Revista Latinoamericana de Educación Inclusiva, 5(2), 153-170.

Echeita, G. (2012). Competencias esenciales en la formación inicial de un profesorado inclusivo. Un proyecto de la agencia europea para el desarrollo de las necesidades educativas especiales. Tendencias pedagógicas, 19, 7-24.

European Commission. (2017). Preparing teachers for diversity: The role of initial teacher education. Bruselas: European Union.

Florian, L. y Black-Hawkins, K. (2011). Exploring inclusive pedagogy. British Educational Research Journal, 37(5), 813-828. https://doi.org/10.1080/01411926.2010.501096

Florian, L. y Spratt, J. (2013). Enacting inclusion: A framework for interrogating inclusive practice. European Journal of Special Needs Education, 28(2), 119-135. https://doi.org/10.1080/08856257.2013.778111

González-Gil, F., Martin-Pastor, E., Poy, R. y Jenaro, C. (2016). Percepciones del profesorado sobre la inclusión: Estudio preliminar. Revista Electrónica Interuniversitaria de Formación del Profesorado, 19(3), 11-24. https://doi.org/10.6018/reifop.19.3.219321

Hirmas C. y Ramos L. (2013). El viaje hacia la diferencia: la escuela inclusiva. Ciudad de México: SM.

Mesa Técnica Educación Especial. (2015). Propuesta para avanzar hacia un sistema educativo inclusivo en Chile: un aporte desde la educación especial. Santiago de Chile: Ministerio de Educación de Chile.

Ministerio de Educación. (2009a). Ley General de Educación $N^{\circ}$ 20370. Gobierno de Chile. Santiago de Chile.

Ministerio de Educación. (2009b). Decreto Supremo 170. Fija Normas para determinar alumnos con necesidades educativas especiales que serán beneficiarios de las subvenciones para Educación Especial. Santiago de Chile.

Ministerio de Educación. (2015a). Ley Núm. 20.845. De inclusión escolar que regula la admisión de los/as estudiantes, elimina el financiamiento compartido y prohíbe el lucro en establecimientos educacionales que reciben aportes del estado. Santiago de Chile.

Ministerio de Educación. (2015b). Diversificación de la enseñanza. Decreto 83. Recuperado de http://especial.mineduc.cl/wp-content/uploads/sites/31/2016/08/Decreto-83-2015.pdf

Ministerio de Educación. (2018). Decreto Supremo 67 de Evaluación, Calificación y Promoción Escolar. Santiago de Chile.

Organización de las Naciones Unidas. (2006). Convención sobre los derechos de las personas con discapacidad. Recuperado de http://www.un.org/esa/socdev/enable/documents/tccconvs.pdf 
Tenorio, S. (2011). Formación inicial docente y necesidades educativas especiales. Estudios Pedagógicos, 37(2), 249-265. https://doi.org/10.4067/So718-07052011000200015

UNESCO. (1990). Declaración mundial sobre educación para todos y el marco de acción para satisfacer las necesidades básicas de aprendizaje. París: UNESCO.

UNESCO. (1994). Declaración sobre necesidades educativas especiales: Acceso y calidad de Salamanca. París: UNESCO.

\section{Breve CV de las autoras}

\section{Cynthia Duk}

Profesora de Educación Diferencial. Master en Integración de Personas con Discapacidad por la Universidad de Salamanca y Magíster en Docencia para la Educación Superior, Universidad Central de Chile. Directora del Centro de Desarrollo e Innovación en Educación Inclusiva y del programa de Magíster en Educación Inclusiva de la Universidad Central. Además, es editora de la Revista Latinoamericana de Educación Inclusiva que publica la Facultad de Educación en colaboración con la Red Iberoamericana de Investigación RINACE. Su línea principal de desarrollo e investigación es la inclusión y la diversidad en educación, con especial énfasis en el desarrollo de escuelas inclusivas. ORCID ID: http://orcid.org/0000-0002-8443-0707 Email: cduk@ucentral.cl

\section{Tatiana Cisternas}

Profesora de Educación Diferencial y Doctora en Ciencias de la Educación. Académica e investigadora actualmente dirige la carrera de Pedagogía en Educación Diferencial de la Universidad Alberto Hurtado. Cuenta con experiencia en formación de profesores en temáticas vinculadas a la educación inclusiva y la enseñanza en contextos de diversidad. A nivel de postgrado desarrolla formación en investigación cualitativa. Ha colaborado en diversas instancias de políticas públicas para la formación docente en inclusión. Sus líneas de investigación abarcan los procesos de enseñanza-aprendizaje, inclusión en la escuela y la formación inicial, inserción y desarrollo profesional de docentes. ORCID ID: https:// orcid.org/0000-0003-3142-0027. Email: tcistern@uahurtado.cl

\section{Liliana Ramos}

Profesora de Educación Diferencial y Magíster en Antropología y Desarrollo. Dirige la carrera de Pedagogía en Educación Diferencial con mención en Desarrollo Cognitivo de la Universidad Diego Portales. Posee una amplia experiencia profesional en el ámbito de la integración e inclusión escolar de estudiantes con necesidades educativas especiales, y en la formación de profesores en temáticas de diversidad, políticas educativas y educación especial. En los últimos años ha dirigido dos convenios en colaboración con la Unidad de Educación Especial de MINEDUC, sobre la implementación del Decreto 83 y la elaboración de las Progresiones de Aprendizaje en Espiral. ORCID ID: https://orcid.org/0000-0001-6802-8083.Email: liliana.ramos@udp.cl 